\title{
LES MYSTÈRES ENTOURANT LA MALADIE DE KAWASAKI: UNE REVUE DE LITTÉRATURE
}

\section{ARTICLE ORIGINAL}

ROSSI, Karoline ${ }^{1}$, MOREIRA, Danilo José Silva ${ }^{2}$, FONSECA, Juliana Brito da ${ }^{3}$, VASCONCELOS, Suzana dos Santos ${ }^{4}$, OLIVEIRA, Vinicius Faustino Lima de ${ }^{5}$, DIAS, Claudio Alberto Gellis de Mattos ${ }^{6}$, OLIVEIRA, Euzébio de ${ }^{7}$, DENDASCK, Carla Viana $^{8}$, ARAÚJO, Maria Helena Mendonça de ${ }^{9}$, FECURY, Amanda Alves ${ }^{10}$

ROSSI, Karoline. Et al. Les mystères entourant la maladie de Kawasaki: une revue de littérature. Revista Científica Multidisciplinar Núcleo do Conhecimento. An 06, Ed. 04, Vol. 05, pp. 52-64. Avril. ISSN: 2448-0959, Lien d'accès: https://www.nucleodoconhecimento.com.br/sante/maladie-de-kawasaki, $\quad$ DOI: 10.32749/nucleodoconhecimento.com.br/sante/maladie-de-kawasaki

\section{RÉSUMÉ}

La maladie de Kawasaki (DK) ou syndrome des ganglions lymphatiques mucocutanés est une vascularite systémique, qui touche principalement les enfants de moins de cinq ans d'origine asiatique, mais peut également atteindre d'autres groupes d'âge, ainsi que toute autre race. L'image clinique de DK a trois étapes :

\footnotetext{
${ }^{1}$ Universitaire en médecine. I'Université fédérale d'Amapá (UNIFAP).

${ }^{2}$ Médecin spécialiste. I'Université fédérale d'Amapá (UNIFAP).

${ }^{3}$ Universitaire en médecine. l'Université fédérale d'Amapá (UNIFAP).

${ }^{4}$ Universitaire en médecine. I'Université fédérale d'Amapá (UNIFAP).

${ }^{5}$ Médecin spécialiste. I'Université fédérale d'Amapá (UNIFAP).

${ }^{6}$ Biologiste, Ph.D. en théorie et recherche comportementale, professeur et chercheur du degree course en chimie de l'Institut d'éducation fondamentale, technique et technologique de l'Amapá (IFAP).

${ }^{7}$ Biologiste, Ph.D. en maladies tropicales, professeur et chercheur du cours d'éducation physique, Université fédérale du Pará (UFPA).

${ }^{8}$ Théologien, Docteur en psychanalyse, chercheur au Center for Research and Advanced Studies (CEPA).

${ }^{9}$ Docteur, Master en enseignement et sciences de la santé, professeur et chercheur du cours de médecine du campus de Macapá, Université fédérale d'Amapá (UNIFAP).

${ }^{10}$ Biomédical, PhD en maladies tropicales, professeur et chercheur du cours médical du campus de Macapá, Université fédérale d’Amapá (UNIFAP).
}

RC: 82506

Disponível em: https://www.nucleodoconhecimento.com.br/sante/maladie-de- 
stade fébrile aigu, dans lequel la congestion conjonctivale, la mucosite orale, l'érythème, l'écaillé, l'éruption polymorphe et la lymphadenopathy laterocervical, apparaissent comme symptômes principaux ; le stade subaigu, qui se produit à la fin de la fièvre, et conduit à l'apparition de l'écaillage de la peau dans les membres, l'arthrite, l'arthralgie et la thrombocytose et enfin le stade de convalescence qui se pose lorsque les symptômes sont presque dissipating et se poursuit jusqu'à leur normalisation. Le traitement le plus utilisé se produit de l'administration de l'immunoglobuline intraveineuse, qui pour un meilleur pronostic de la pathologie devrait être initié tôt.

Mots-clés: Syndrome des ganglions lymphatiques mucocutanés, Vasculite, Artérite, Fièvre, Kawasaki.

\section{INTRODUCTION}

La maladie de Kawasaki (DK) est une vascularite systémique qui affecte les vaisseaux de petit et moyen calibre et touche principalement les enfants de moins de 5 ans, étant l'une des raisons des maladies cardiaques pendant l'enfance (HUANG et al., 2015; MAGALHÃES, 2008). L'étiologie de DK, c'est-à-dire les agents de causalité de cette maladie, ne sont pas connues. Cependant, certains aspects cliniques et épidémiologiques proposent des raisons infectieuses possibles (FERRONATO et coll., 2010).

KD est la deuxième vascularite la plus commune dans l'âge pédiatrique, affectant principalement des enfants moins d'une demi-décennie (90\%). II est rare chez les enfants de moins de 6 mois et plus de 8 ans, qui, cependant, sont plus enclins au développement des anévrismes coronaires. À l'échelle mondiale, l'occurrence est différente, le Japon étant le pays le plus touché, avec une incidence annuelle de 110 à 150 cas pour 100000 enfants de moins de 5 ans. Compte tenu du calcul des taux d'incidence chez 100000 enfants de moins de 5 ans, aux États-Unis d'Amérique (USA) DK est la cause la plus fréquente de maladies cardiaques acquises par

RC: 82506

Disponível em: https://www.nucleodoconhecimento.com.br/sante/maladie-dekawasaki 
l'enfance, allant de 9,1 à 32,5 cas, se produisant plus fréquemment chez ceux des descendants d'îles asiatiques et pacifiques $(32,5)$, intermédiaires chez les AfroAméricains $(16,9)$ et hispaniques $(11,1)$ et rares chez les Caucasiens $(9,1)$ (ALMEIDA et al., 2010; CASTRO; URBANO; COSTA, 2009; RODRIGUES et al, 2017).

DK a une prévalence plus élevée dans la population asiatique, en mettant l'accent sur le japonais, affectant principalement les enfants. II y a un rapport d'une incidence annuelle au Japon et en Corée de jusqu'à 100 cas pour 100.000 habitants, alors que dans la course caucasienne l'occurrence est entre 6 à 10 cas pour 100.000 habitants sous l'âge d'une demi-décennie. En Amérique latine, on estime qu'il y a 3 cas pour 100000 habitants, ce qui explique une fréquence plus faible de cette pathologie dans les populations non asiatiques qui ne descendent pas d'Asiatiques (SCARDINA et al., 2007). En ce qui concerne le taux de mortalité associé au DK, il est très faible, car il est inférieur à 0,1 \% (BARCA et coll., 2019).

L'incidence de la DK varie géographiquement dans le monde, étant plus répandue chez les descendants japonais, suggérant une prédisposition génétique de la maladie, en plus d'agents causatous possibles qui peuvent être liés à l'emplacement géographique, tels que les bactéries (CASTRO; URBANO; COSTA, 2009; RODRIGUES et al, 2017).

La variation saisonnière de l'incidence de la DK est bien connue, mais les taux changent d'un pays à l'autre. Au Royaume-Uni, en Australie et aux États-Unis, le nombre de cas est plus élevé en hiver et au printemps. En Chine et en Corée, au printemps et en été. Aux États-Unis et au Royaume-Uni, la hausse des taux est plus corrélée avec la variation des périodes pluvieuses qu'avec la variation de température. Au Brésil, une étude menée auprès de 70 patients atteints de DK a montré un dossier plus élevé de la maladie coïncidant avec des périodes d'incidence plus élevée de maladies infectieuses de mai à juin (début de la sécheresse) et novembre et décembre (pluies précoces) (MAGALHÃES; ALVES, 2017).

RC: 82506

Disponível em: https://www.nucleodoconhecimento.com.br/sante/maladie-dekawasaki 
Une personne atteinte de DK développe des caractéristiques symptomatologiques qui mènent à la présentation d'une image clinique, qui se compose de l'ensemble des signes et des symptômes manifestés par le patient (KAYIRAN ; DINDAR; GURAKAN, 2010). Ainsi, l'image clinique de DK est caractérisée par lymphadenopathy cervical, fièvre, amygdalite, gonflement des membres, éruption, démangeaisons et écaillage (ATIK, 2007; SCARDINA et al., 2007). En outre, DK peut causer l'inflammation généralisée, affectant quelques organes causant la cardiomyopathie inflammatoire, la méningite stérile, l'hépatite, l'adénolinefite, la péricardite, et l'angite (CASTRO; URBANO; COSTA, 2009).

Castro, Urbano et Costa (2009) soulignent une relation entre la vascularite causée par DK et l'implication de certains organes, qui consistent en un regroupement des tissus responsables du maintien de l'organisme.

DK a été rapporté pour la première fois en 1967 par le médecin japonais Tomisaku Kawasaki, qui l'a défini comme " syndrome des ganglions lymphatiques mucocutanés ». Au cours de cette période, 50 cas ont été signalés entre 1961 et 1967. Au Japon, l'occurrence est plus élevée, et la distribution mondiale a sa prévalence variable (ALMEIDA et al., 2010; SCARDINA et al., 2007; MAGALHÃES; ALVES, 2017).

Dans le scénario actuel, certaines données épidémiologiques sur la DK ont déjà été établies, permettant une analyse plus précise des facteurs qui interviennent dans la diffusion et la propagation des maladies. De nombreuses études ont été menées afin de comprendre la pathophysiologie de la maladie, qui consiste en un ensemble de changements anormaux causés dans le corps (CASTRO; URBANO; COSTA, 2009).

Dans une étude, des preuves de DK et de l'avance d'autres comorbidités ont été trouvées, qui se rapporte au fait que deux maladies ou plus sont en même temps dans un individu, ainsi les complications de DK seraient l'évolution défavorable de cette association (SCARDINA et al., 2007).

RC: 82506

Disponível em: https://www.nucleodoconhecimento.com.br/sante/maladie-dekawasaki 
Pour améliorer les soins et la qualité de vie du patient atteint de DK, des traitements ont été mis au point, qui consistent en des façons de prendre soin d'un patient (CASTRO; URBANO; COSTA, 2009).

\section{OBJECTIFS}

Détailler les agents causatous possibles, la pathophysiologie, les organes principaux et les structures anatomiques affectées par la maladie de Kawasaki (DK).

Vérifier les principales caractéristiques de l'image clinique d'une personne pour le diagnostic, les principales complications associées, et les traitements disponibles pour les patients atteints de DK.

\section{MÉTHODE}

II s'agit d'une recherche bibliographique menée dans les bases de données, Scielo, Bibliothèque numérique brésilienne des thèses et mémoires, LILACS et PubMed, à l'aide de mots clés ou de leurs associations : maladie de Kawasaki-Kawasaki.

Les paramètres d'inclusion utilisés dans la préparation des recherches étaient la disponibilité complète en ligne, l'approche directe sur la maladie de Kawasaki (DK) ou sur certains aspects pertinents à cette maladie. Dans la base de données Scielo, il y avait des filtres texte des productions écrites en anglais et en portugais, dans les autres bases de données ce critère n'a pas été utilisé. Les paramètres d'exclusion appliqués ont été dupliqués et exécutés avant l'an 2000.

Une analyse a été faite dans les bases de données mentionnées pour énumérer les articles liés à DK dans la littérature. D'après une lecture antérieure des titres et des résumés des productions scientifiques trouvées, les études qui ne correspondaient pas à ce qui devait constituer cet examen ont été écartées. Les textes complets qui

RC: 82506

Disponível em: https://www.nucleodoconhecimento.com.br/sante/maladie-dekawasaki 
passaient par les étapes précédentes ont été lus, sélectionnant pour cet examen ceux qui abordaient les aspects pertinents de DK.

\section{RÉSULTATS}

\section{AGENTS CAUSATOUS POSSIBLES DE DK}

La cause de la maladie de Kawasaki est encore inconnue. Cependant, ses caractéristiques cliniques et épidémiologiques alimentent l'hypothèse qu'un agent infectieux est la cause déclenchante, qui produit des changements dans les cellules, les tissus et les organes des individus avec la prédisposition génétique. Cette hypothèse est la plus probable, mais elle n'a toujours aucune preuve (CASTRO; URBANO; COSTA, 2009).

Une des théories est celle du coronavirus NL-63, détectée au moyen d'une réaction en chaîne de polymérase (PCR) dans les fluides de voie aérienne de 11 patients présentant KD et dans seulement 1 des 22 patients de contrôle. Cependant, 5 autres études ultérieures n'ont pas trouvé de coronavirus dans des échantillons de tissus respiratoires ou nasopharites prélevés sur des patients, ce qui rend impossible pour ce virus d'être la cause déclenchante (CASTRO; URBANO; COSTA, 2009).

Une autre théorie est celle de la stimulation immunologique par les superantigènes bactériens tels que les toxines staphylococciques et streptococciques. Une troisième théorie est immunologique, qui propose que la DK soit causée par une réponse immunitaire aberrante chez les individus génétiquement prédisposés (MAGALHÃES; ALVES, 2017).

\section{PHYSIOPATHOLOGIE DE DK}

Les altérations trouvées dans DK, particulièrement histologiques, caractérisent une image de l'angiite systémique généralisée, affectant la plupart du temps des navires

RC: 82506

Disponível em: https://www.nucleodoconhecimento.com.br/sante/maladie-dekawasaki 
de calibre médian, particulièrement les artères coronaires. Dans plusieurs organes, des changements infâmes peuvent être observés, comme dans le myocarde, dans le péricarde, dans les vaisseaux sanguins, dans les méninges, dans le poumon, dans les ganglions lymphatiques et dans le foie (CASTRO; URBANO; COSTA, 2009).

\section{PRINCIPAUX ORGANES ANATOMIQUES ET STRUCTURES AFFECTÉS PAR LE DK}

DK affecte les organes et les artères, principalement les vaisseaux de calibre moyen de divers systèmes du corps humain, tels que les maladies cardiovasculaires, pulmonaires, gastro-intestinales (intestin et vésicule biliaire sont les principaux organes affectés) et le système nerveux central (SNC). Cependant, il convient de souligner l'implication que cette maladie a dans le cœur, avec la formation d'anévrismes coronaires (CASTRO; URBANO; COSTA, 2009).

D'autres artères peuvent également être affectées, telles que : anévrisme aortique, avec un plus grand nombre de cas rapportés compromettant l'aorte abdominale ; anévrisme d'artère axillaire ; anévrisme de l'artère brachiocéphale; anévrismes d'artères iliaques, fémorales; anévrisme de l'artère rénale et obstruction oculaire de l'artère (ALVES et al., 2011).

\section{PRINCIPALES CARACTÉRISTIQUES DE L'IMAGE CLINIQUE D'UN INDIVIDU POUR LE DIAGNOSTIC DE DK}

La fièvre, qui marque la phase aiguë de la maladie, est généralement élevée (audessus de $39{ }^{\circ} \mathrm{C}$ ) et aux sommets, diminuant temporairement en intensité d'environ sept à quatorze jours, et peut atteindre trois et/ou quatre semaines s'il n'y a pas de traitement approprié. Le jour où la fièvre commence est considéré comme le premier jour de la maladie. Cependant, quelques patients ont d'autres manifestations classiques avant fièvre, telles que : conjonctivite non purulent ; ganglion lymphatique cervical; érythèmes et/ou œdèmes sur les mains et les pieds; escalatiniforme

RC: 82506

Disponível em: https://www.nucleodoconhecimento.com.br/sante/maladie-dekawasaki 
exanthetem, morbiliforme ou polymorphe; langue framboesiforme, érythème et œdème d'oropharynx, fissures et érythème labial (CASTRO; URBANO; COSTA, 2009).

Les changements dans les extrémités (mains et pieds) avec les érythèmes et/ou l'oedème apparaissent également dans la phase aiguë, et l'oedème est très douloureux et est associé à l'érythème palmoplantaire avec la vascularite au bout des doigts qui après une période d'une à deux semaines, déjà dans la phase subacute, commence à peler, commençant par la région périungueal dans le doigt de gant (MAGALHÃES ; ALVES, 2017).

La conjonctivite bilatérale non exsudative affecte particulièrement la conjonctive bulbaire par rapport à la conjonctive palpebrale et tarsale et est indolore. Parfois, il est accompagné d'iridocyclite, une inflammation de la partie antérieure de l'œil, avec une résolution rapide et peu associée à la sensibilité à la lumière (CASTRO; URBANO; COSTA, 2009).

L'éruption polymorphe est un ensemble d'éruptions cutanées qui se produisent dans les premiers jours de la maladie, affectant principalement le tronc et les extrémités, sans cloques apparentes ou vésicules. Ils ont une présentation variable (éruption urticariforme, morbiliforme, maculopapular) ou diffuse, ressemblant à la scarlatine (ALMEIDA et al., 2010).

Changements dans les lèvres et la cavité buccale 9 cas sur 10 dans la phase aiguë avec hyperémie labiale intense et oropharynx, fissures, muqueuse sèche et saignement des lèvres. Les papilles de langue peuvent devenir proéminentes et des ulcérations orales avec l'exudato d'oropharynx peuvent parfois être observées. En ce qui concerne la lymphadenomegaly cervicale, un autre critère diagnostique, le patient doit présenter au moins un ganglion lymphatique avec un diamètre supérieur ou égal à $1,5 \mathrm{~cm}$, généralement unilatéral, douloureux et ferme, dans le triangle cervical antérieur (ALMEIDA et al., 2010; MAGALHÃES; ALVES, 2017).

RC: 82506

Disponível em: https://www.nucleodoconhecimento.com.br/sante/maladie-dekawasaki 


\section{LES PRINCIPALES COMPLICATIONS ASSOCIÉES À DK}

Chez les patients atteints de DK, trois types de vasculopathies sont identifiés à différents moments de l'évolution de la maladie : artérite nécrosante, artérite subaiguë/chronique et vascularite de prolifération myofibroblastique luminale. Dans la phase aiguë de la maladie, l'artérite nécrosante se distingue, qui a son processus pathologique complet dans les deux premières semaines après le début de l'état fébrile, en particulier dans les artères coronaires, qui peuvent déterminer la formation d'anévrismes géants sensibles au développement de la thrombose et des ruptures. Dans la phase subacute, il est vérifié que le processus de vascularite subaiguë commence 2 semaines après le début de la fièvre, et peut affecter tous les vaisseaux sanguins, en particulier le segment moyen des artères musculaires moyennes, telles que les artères coronaires. Dans la phase chronique de la maladie, la myoprolifération fibroblastique luminale se produit, associée à la vascularite subaiguë chronique (MAGALHÃES; ALVES, 2017).

La complication la plus grave de la maladie est la vascularite coronaire, avec des changements dans les artères coronaires, qui affecte $15-20 \%$ des patients qui ne reçoivent pas de traitement. Ces altérations comprennent les anévrismes, les ectasias et les sténoses des artères coronaires, responsables de la mortalité de $2 \%$ (ALMEIDA et al., 2010).

Dans le système gastro-intestinal, il y a des vomissements, de la diarrhée, des douleurs abdominales et des ileus paralytiques. Au début de la fièvre, les manifestations les plus fréquentes sont les vomissements et la diarrhée et les moins fréquentes sont l'ictère, l'hépatomegalie douloureuse, la pancréatite et les vésicules hydropic. Dans le système respiratoire, les manifestations les plus fréquentes sont la toux, la pneumonite et les changements radiologiques. Se produit rarement rinorreia (MAGALHÃES; ALVES, 2017).

RC: 82506

Disponível em: https://www.nucleodoconhecimento.com.br/sante/maladie-dekawasaki 
Dans le SNC, la maladie peut se manifester par une irritabilité extrême dans la phase aiguë, en plus de la méningite aseptique, de l'ataxie, de la paralysie faciale et de la perte auditive neurosensorielle. II convient également de noter la présence de ganglionite et de névrite dans les nerfs crâniens et périphériques, l'endarteritis, la périarteritis, la chorioméningitis et la leptomeningitis, en plus de l'atrophie, de la dégénérescence avec perte de neurones, du gliosis marginal et subpendimal et de la formation des nodules gliales autour des dégénérescences de neurones (MAGALHÃES; ALVES, 2017).

Des changements comportementaux tels que la déficience de l'attention, la déficience d'apprentissage, les changements émotionnels (labilité émotionnelle, peur et terreur nocturne) et les problèmes d'internalisation (comportement anxieux, dépressif et agressif) ont été décrits dans la littérature (ALVES et al., 2011).

\section{TRAITEMENTS DISPONIBLES POUR LES PERSONNES TOUCHÉES PAR DK}

La thérapie pour DK a deux buts différents selon l'étape dans laquelle elle est située. Dans la phase aiguë, il vise à réduire la réponse inflammatoire dans la paroi coronarienne et à prévenir la vascularite et ses conséquences (thrombose et anévrismes). Dans les phases subaiguës et chroniques, l'objectif est de prévenir l'ischémie et l'infarctus du myocarde (CASTRO; URBANO; COSTA, 2009).

L'immunoglobuline intraveineuse (IGEV) est le principal médicament utilisé en DK et son mécanisme d'action reste inconnu. II est utilisé dans la phase aiguë, de préférence dans les 7 à 10 premiers jours du début de la condition (MAGALHÃES; ALVES, 2017).

Le traitement consiste en l'administration d'immunoglobuline en une seule dose de $2 \mathrm{~g} / \mathrm{kg}$ sur une période variable de 10 à 12 heures ainsi que l'administration d'acide acétylsalicylique (AAS) 80 à $100 \mathrm{mg} / \mathrm{kg} / \mathrm{jour}$, oralement, 6/6 heures. L'AAS à haute

RC: 82506

Disponível em: https://www.nucleodoconhecimento.com.br/sante/maladie-dekawasaki 
dose a un effet anti-inflammatoire et, à faibles doses, agit comme inhibiteur de l'agrégation plaquettaire, de sorte que le temps d'utilisation de l'AAS à fortes doses devrait rester jusqu'à ce que l'enfant soit afébrile. Ensuite, la dose est réduite à 3 à 5 $\mathrm{mg} / \mathrm{kg} / \mathrm{jour}$ et maintenue à cette dose tandis que le patient se présente avec la thrombocytose et/ou les changements coronaires (MAGALHÃES; ALVES, 2017; RODRIGUES et al., 2017).

Entre 1 et 2 patients sur 10 atteints de DK ne répondent pas à la première dose d'IGEV, ou avec la permanence de l'état fébrile ou avec la réapparition de celui-ci un jour et demi après la première perfusion. Ces patients sont considérés comme réfractaires au traitement et sont ceux qui ont le plus grand risque de changements coronariens. Dans de tels cas, l'administration de la deuxième dose d'IGEV de 2 g/kg en une seule dose est recommandée. Si la fièvre persiste pendant 36 heures, il est recommandé d'utiliser la thérapie pulsée avec de la méthylprednisolone à une dose de $30 \mathrm{mg} / \mathrm{kg} / \mathrm{jour}$ (dose maximale de $1 \mathrm{~g} / \mathrm{jour}$ ), infusée à 1 heure, 1 heure/jour, pendant 3 jours (MAGALHÃES; ALVES, 2017).

Dans le traitement de la DK dans les phases subaiguës et chroniques, l'aspirine à faible dose (3-5mg/kg/jour) est utilisée chez les enfants atteints d'anévrismes petits et moyens. Dans ces phases, le traitement vise à prévenir la thrombose (activation des plaquettes) et la ssthèse des vaisseaux. D'autres agents antiplaquettaires sont également utilisés (clopidogrel, ticlopidine, dipyridamol) et, associés à l'aspirine, se présentent comme plus concrets dans le blocage de l'agrégation plaquettaires (CASTRO; URBANO; COSTA, 2009).

\section{DISCUSSION}

\section{CORRÉLATION ENTRE LES CAUSALITÉS POSSIBLES ET LE DÉVELOPPEMENT DE DK}

RC: 82506

Disponível em: https://www.nucleodoconhecimento.com.br/sante/maladie-de- 
À ce jour, l'origine de DK n'a pas été identifiée, mais il y a quelques agents, qui sont censés être liés au développement de la pathologie. II s'agit de Staphylococcus aureus, estreptococos, vírus influenza, morbilivírus, paramixovírus, bunyavírus. L'adénovirus et un nouveau coronavirus humain appelé coronavirus de New Haven (Nco-NH) peuvent également être associés à la $\mathrm{DK}$, car ils ont déjà été identifiés dans les sécrétions des voies respiratoires des patients atteints de la maladie. Ces virus et bactéries pourraient agir sur le développement de la DM, de sorte qu'ils conduiraient à des données causées par la maladie, telles que les anévrismes aortiques (BARCA et al., 2019; CASTRO; URBANO; COSTA, 2009).

II y a une hypothèse qui associe l'existence des superantigènes bactériens au développement de DK. Les études analysant cette suggestion supposent que le superantigène peut se lier à la cellule antigène-présentation, par le complexe majeur d'histocompatibilité de classe II (MHC de classe II) et le récepteur de lymphocyte de $T$ aux emplacements contraignants différents de ceux des antigènes communs. Ceci favoriserait une réponse immunologique avec la formation et la libération des cytokines pro-inflammatoires dans les concentrations excessives, contribuant à l'évolution d'un processus inflammatoire caractéristique de DK (CASTRO ; URBAIN; COAST, 2009).

Quelques indications mènent au cru qu'une réponse oligoclonale d'IgA se produit dans DK aigu. Une étude menée par Castro, Urbano et Costa (2009) a effectué l'analyse de la chaîne alpha lourde d'immunoglobulines recueillies à partir d'une grande quantité de leucocytes accumulés à un site de réponse inflammatoire de la paroi artérielle des patients atteints de DK, qui a montré que des anticorps spécifiques d'immunoglobuline A ( $\lg A)$ ont été détectés dans l'échantillon. Cela témoigne de l'existence d'une réponse immunitaire liée à DK (CASTRO; URBANO; COSTA, 2009).

L'hypothèse selon laquelle le DK a une prédisposition génétique est renforcée par l'incidence élevée chez les Asiatiques et leurs rummies. Une étude a montré 67

RC: 82506

Disponível em: https://www.nucleodoconhecimento.com.br/sante/maladie-dekawasaki 
gènes liés au développement de la pathologie, qui impliquait la participation à l'endothélium, le métabolisme lipidique, l'activation des mécanismes immunologiques et le recrutement de plaquettes, soutenant l'idée qu'il existe une relation génétique pour le développement de DK (CASTRO; URBANO; COSTA, 2009; SOTELO; GONZÁLEZ, 2007).

\section{PHYSIOPATHOLOGIE DE DK}

La vascularite commence par l'apparition d'un gonflement et d'une inflammation, qui peuvent survenir pendant une période variable. Les neutrophiles sont présents au début, et peu de temps après il y a une prédominance des lymphocytes $T$ cytotoxiques CD8+ et de l'immunoglobuline d'IgA (CASTRO; URBANO; COSTA, 2009).

Dans les artères coronaires il y a des changements inflammatoires avec la présence de l'oedème et de la nécrose des cellules musculaires. Avec cette perte d'intégrité structurelle, il y a la formation d'anévrismes. En outre, les fibroblastes prolifèrent puis se transforment, conduisant à la présence possible de susthèse, calcifications et thrombose (CASTRO; URBANO; COSTA, 2009; SOTELO; GONZÁLEZ, 2007).

Il est important de noter que le facteur inflammatoire 1 trouvé dans les allogreffes est fortement augmenté dans les tissus artériaux shimnotic de DK. Ce facteur inflammatoire 1 de l'allogreffe lie la réponse de type I d'interféron jusqu'à l'activation des macrophages spécifiques et des lymphocytes $T$, qui assume l'importance probable de l'interférence de ces cellules dans l'artérite par DK (ROWLEY et autres, 2017).

\section{COMMENT LES ORGANES PRINCIPAUX ET LES STRUCTURES ANATOMIQUES SONT AFFECTÉS PAR DK}

RC: 82506

Disponível em: https://www.nucleodoconhecimento.com.br/sante/maladie-de- 
DK affecte les organes et les artères, principalement les vaisseaux de taille moyenne, tels que ceux du cœur, en particulier les artères coronaires, avec la formation d'anévrismes. La maladie peut également affecter le système respiratoire (poumon), par vascularite, le système gastro-intestinal, principalement l'intestin et la vésicule biliaire, et le système nerveux (CASTRO; URBANO; COSTA, 2009).

Le système lymphatique peut être affecté par kd, car l'un des symptômes de DK est gonflement des ganglions lymphatiques cervicaux. Le système cutané est endommagé par un érythème cutané, suivi d'œdèmes. Dans les yeux, l'hyperémie non suputive de conjoncture peut se produire et les muqueuses de la cavité buccale peuvent être affectées par la mucosite orale et les lésions sur les lèvres (SOTELO; GONZÁLEZ, 2007).

\section{LES PRINCIPALES CARACTÉRISTIQUES DE L'IMAGE CLINIQUE D’UNE PERSONNE AVEC DK ET COMMENT ILS L'AFFECTENT}

Le cours clinique de DK peut être divisé en trois étapes cliniques : aiguë, subaiguë et convalescence. Le stade fébrile aigu dure habituellement de 7 à 14 jours, caractérisé par la congestion conjonctivale, la mucosite orale, l'érythème, l'écaillé et l'éruption polymorphe et la lymphadenopathie. Le stade subacute est la période entre la fin de la fièvre et le 25ème jour de la maladie. À ce stade, les patients se présentent avec l'écaurage de peau dans les membres, l'arthrite, l'arthralgie et la thrombocytose. Le stade de convalescence commence lorsque les symptômes cliniques commencent à disparaître et se poursuit jusqu'à l'établissement normal de I'VHS (taux de pression artérielle rouge), qui est habituellement observé 6 à 8 semaines après l'apparition des symptômes. Ces étapes affectent la vie du patient, puisqu'elles l'empêchent d'exécuter ses activités quotidiennes, qui sont principalement dues aux lésions de peau et à la symptomatologie (ALMEIDA, 2017; SCARDINA et al., 2007).

La condition suggestive de la pathologie est caractérisée par la fièvre, les ganglions lymphatiques cervicals accrus et l'amygdalite, suivie de l'érythème cutané

RC: 82506

Disponível em: https://www.nucleodoconhecimento.com.br/sante/maladie-dekawasaki 
généralisée, des démangeaisons et de l'oedème des membres et après quelques jours d'écaillage. Les essais en laboratoire montrent une phase inflammatoire active avec un taux élevé de sédimentation, une protéine $C$ réactive, une leucocytose et un nombre accru de plaquettes, ce qui conduit à la léthargie de l'individu affecté, puisque l'inflammation peut causer de fortes douleurs (ALMEIDA, 2018; ATIK; FORONDA; BUSTAMANTE, 2003; FERRONATO et al., 2010; ROSSI et al., 2015).

L'hyperémie non suputive de conjoncture peut également surgir ; éruption maculairepapular ; lésions sur les lèvres et la cavité buccale; changements dans la peau des extrémités, en plus du murmure précordial, difficulté à respirer, douleurabdominale, dysurie, hépatomegaly, arthralgie, raidissement du cou et convulsions. Ces symptômes empêchent le patient de voir et de se déplacer normalement, puisque les yeux sont endommagés et la douleur cause des difficultés, d'effectuer des mouvements (CASTRO; URBANO; COSTA, 2009; SOTELO; GONZÁLEZ, 2007).

$\mathrm{KD}$ affecte plusieurs patients atteints de vascularite dans divers organes, comme cela a été dit plus tôt, le poumon, l'intestin, la vésicule biliaire, le système nerveux central, sont des exemples, ce qui conduit à l'apparition de symptômes tels que la fatigue, la perte de poids et la douleur dans les articulations et les muscles, mais les dommages cardiaques est le plus important, avec la formation d'anévrismes coronaires, qui conduisent à l'angine de poitrine (CASTRO; URBANO; COSTA, 2009; PRINTZ, 2011).

Un autre symptôme est l'irritation, qui apparaît chez les enfants atteints de la maladie, et les plaintes gastro-intestinales, y compris la diarrhée, les vomissements et les douleurs abdominales, surviennent chez environ un tiers des patients, ce qui explique le fait que les enfants atteints de DK sont plus irrités que les autres (ALMEIDA, 2018; KAYIRAN; DINDAR; GURAKAN, 2010).

L'abcès et l'oedème rétropharyngéal sont habituellement associés à une condition mortelle s'ils ne sont pas traités correctement. Les otorhinolaryngologistes peuvent le

RC: 82506

Disponível em: https://www.nucleodoconhecimento.com.br/sante/maladie-dekawasaki 
traiter avec le drainage chirurgical de l'abcès, empêchant le bloc de voie aérienne (KIM; KWON, 2016; XIE et al., 2018).

La mucosite orale est représentée par l'érythème diffus de la muqueuse, la rougeur dans les lèvres et la langue, et l'hypertrophie des papilles linguales avec le développement ultérieur de la langue de fraise, et se produit habituellement au stade aigu de la maladie et de la convalescence, en conséquence du traitement pharmacologique, et empêche le patient de se nourrir correctement en raison des lésions, affectant sa nutrition proportionné (SCARDINA et al., 2007).

\section{LE DIAGNOSTIC DE DK}

DK représente un défi à diagnostiquer tôt, car il s'agit d'un trouble rhumatologique inhabituel qui nécessite une intervention spécifique afin d'éviter les séquelles graves ou mortelles (ALMEIDA et al., 2010).

La clinique de la maladie n'est pas claire, étant commun le diagnostic initial d'autres maladies, telles que la scarlatine, l'exanthème viral ou les conditions allergiques, dues aux symptômes initiaux de la maladie. Même avec l'accomplissement complet des critères, il est fréquent de retarder dans le diagnostic correct, qui mène à un retard dans le début du traitement approprié, favorisant l'aspect des complications, qui peuvent rapidement aggraver l'état clinique du patient. En outre, il existe des formes incomplètes ou atypiques, qui rendent le diagnostic encore plus difficile. Le diagnostic tôt de la maladie est important, car le traitement dans la phase aiguë réduit le processus inflammatoire dans les artères coronaires et empêche la thrombose, réduisant les chances de mortalité (FERRONATO et al., 2010).

Le diagnostic clinique opportun est essentiel pour établir ce traitement tôt et éviter des lésions coronaires. Ce type de blessure peut causer l'ischémie ou l'infarctus du myocarde, qui est extrêmement grave (KAYIRAN; DINDAR; GURAKAN, 2010; SOTELO; GONZÁLEZ, 2007).

RC: 82506

Disponível em: https://www.nucleodoconhecimento.com.br/sante/maladie-dekawasaki 
Les critères diagnostiques de la maladie de DK classique sont la fièvre avec cinq jours ou plus, en plus d'au moins quatre autres critères, qui sont : érythème palmoplantaire avec ou sans oedème douloureux ou écaillé périunigueal ; Exathème polymorphe; hyperémie conjonctivale bulbaire des deux côtés, non exsudative ; Changements dans les lèvres et la muqueuse orale; Lymphadénopathie cervicale diamètre supérieur à $1,5 \mathrm{~cm}$, dur et indolore sur palpation. Le diagnostic de DK incomplet peut être établi en présence de trois ou plusieurs des critères suivants : Albumin $\leq 3 \mathrm{~g} / \mathrm{dL}$; Anémie; Élévation de l'aminotransferase d'alanine ; Plaquettes après sept jours $\geq 450000 / \mathrm{mm}^{3}$; Leucocytes $\geq 15000 / \mathrm{mm}^{3}$; Urine $\geq 10$ leucocytes (CASTRO; URBANO; COSTA, 2009; RODRIGUES et al., 2017; YAP; LIN; WANGUI, 2012).

Les séquelles principales de la maladie sont liées au système cardiaque, par conséquent, la formation image cardiaque par échocardiographie est une partie pertinente de l'évaluation des patients présentant le DK suspecté. Pour les cas simples, l'échocardiographie est recommandée au diagnostic, 6 à 8 semaines après le début de la maladie (ATIK et al., 2017; KAYIRAN; DINDAR; GURAKAN, 2010).

Les altérationslaboratoires les plus fréquentes sont le nombre sanguin avec le leukocytosis et la neutrophileia, en plus d'augmenter les valeurs des essais aigus de phase, tels que la vitesse d'hémosedimentation et la protéine $C$ réactive. La maladie n'est souvent diagnostiquée qu'après la participation de l'artère coronaire (FERRONATO et al., 2010).

Une étude de Kentsis et al. (2013) ont constaté que des marqueurs de filamine $\mathrm{C}$ et de meprina A ont été détectés dans le sérum et l'urine des deux groupes indépendants de patients participants de DK. La même étude révèle que l'utilisation de ces marqueurs pour effectuer le diagnostic de DK est plus efficace que l'utilisation des marqueurs actuellement utilisés (KENTSIS et al., 2013).

\section{EXAMENS D'IMAGE ET COMPLÉMENTAIRES}

RC: 82506

Disponível em: https://www.nucleodoconhecimento.com.br/sante/maladie-de- 
Echocardiogramme est l'un des principaux tests utilisés pour le diagnostic de DK, car il n'est pas invasif, a une sensibilité élevée et la spécificité pour analyser et détecter les anomalies dans les artères coronaires, évaluer le myocarde et vérifier la présence de péricardite. Dans cet examen, quelques résultats peuvent sembler qui indiquent l'artérite coronaire, qui précède la formation des anévrismes, tels que la luminosité perivasculaire, l'ectasia ou le rétrécissement des artères coronaires. Dans l'analyse des artères coronaires, le diamètre interne du navire doit être évalué; le nombre, l'emplacement et le type (saccular ou fusiforme) des anévrismes; et la présence ou non de thrombus intraluminal et de susthèse. Dans la phase aiguë de la maladie, quelques résultats fréquents dans des échocardiogrammes sont : réduction de la contractilité gauche de ventricule, régurgitation de la valve mitrale et de l'effusion péricardique (CASTRO; URBANO; COSTA, 2009; MAGALHÃES; ALVES, 2017; RODRIGUES et al., 2017).

Autres tests qui peuvent être effectués, en fonction de la disponibilité, la gravité, les avantages et les indications qui varient selon le cas. Par exemple, imagerie par résonance magnétique (RNM), angiorésonance, radiographie pulmonaire, tomographie calculée haute résolution, angiographie (CASTRO; URBANO; COSTA, 2009).

L'angiographie de CT, un examen invasif qui a des risques, et l'angioresonance sont habituellement exécutés dans ces patients qui ont présenté l'anomalie sur l'électrocardiogramme, l'échocardiographique ou les symptômes de l'ischémie myocardique (CASTRO; URBANO; COSTA, 2009; MAGALHÃES; ALVES, 2017).

Dernièrement, la tomographie calculée à haute résolution a été plus indiquée, surtout par rapport à l'IRM, puisque son exécution est plus rapide et plus facile à interpréter, tandis que l'IRM nécessite un temps anesthésique plus long des enfants pour la collecte (CASTRO; URBANO; COSTA, 2009).

RC: 82506

Disponível em: https://www.nucleodoconhecimento.com.br/sante/maladie-dekawasaki 
La radiographie pulmonaire, qui ne présente généralement pas d'altérations, dans environ $15 \%$ des cas des anomalies sont découvertes, telles que: modèle de reticulogranular ou péribronchial infiltrer, l'accumulation de trop de liquide dans l'espace entre les plèvres viscérales et pariétales ou l'effondrement du poumon. Ils apparaissent après le 10ème jour de la maladie et sont possibles de vérifier les radiographies 10 à 50 jours après le début de la condition (CASTRO; URBANO; COSTA, 2009).

Dans les essais en laboratoire, les résultats ne sont pas spécifiques à la DK, mais plutôt une indication d'un processus inflammatoire élevé et il est possible de trouver, au début de la phase aiguë, une augmentation de la vitesse d'hémosedimentation (VHS), de la protéine $\mathrm{C}$ réactive (PCR) et des taux d'alphaglycoprotéine acide. Dans le test sanguin, il est possible de vérifier une quantité anormalement élevée de neutrophiles, appelée leucocytose avec neutrophile, et peut être accompagnée d'une augmentation des chauves-souris ou de l'éosinophile. En outre, la quantité de plaquettes est habituellement normale dans la phase aiguë, cependant, il est possible que dans certains cas il y ait thrombocytopénie, qui est associée au développement de la maladie coronaire grave et de l'infarctus du myocarde (MAGALHÃES ; ALVES, 2017).

\section{ANALYSE DES PRINCIPALES COMPLICATIONS ASSOCIÉES À DK}

La complication la plus grave de la maladie est la vascularite coronaire, qui affecte $15-20 \%$ des patients non traités, qui peuvent mener aux anévrismes, aux ectasias et à la susthèse des artères coronaires, qui expliquent la mortalité de $2 \%$. D'autres complications de DK ont été décrites comme anévrismes dans d'autres artères telles que l'aorte ; l'aorte abdominale; artère axillaire; artère brachiocéphale; artères iliaques, artères fémorales et artères rénales, ce qui indique comment le DK peut affecter considérablement le corps humain, et donc être extrêmement dangereux

$\mathrm{RC}: 82506$

Disponível em: https://www.nucleodoconhecimento.com.br/sante/maladie-dekawasaki 
(ALVES, et al., 2011; CHBEIR et al., 2018; MUTLUER; ÇELIKER, 2019; PRINTZ, 2011; XIE et al., 2018)

Quelques patients présentant DK sont sensibles à la dilatation coronaire progressive. Une telle dilatation coronaire se produit pendant la phase aiguë et peut procéder à la phase subaiguë, qui aggrave l'état clinique de plusieurs patients (BRIEDÉ et al., 2015; 2015; 2015; LIU et al., 2017).

Les complications gastro-intestinales dans DK peuvent se produire telles que l'obstruction intestinale, l'oedème du côlon, l'ischémie intestinale, la pseudoobstruction intestinale, et l'abdomen aigu, qui affecte le patient alarmant, car ces complications peuvent mener au sepsis et même à la mort. En outre, les altérations ophtalmologiques associées à DK peuvent inclure l'uvéite, l'iridocyclite, l'hémorragie conjonctivale, la nurite optique, l'amaurose, et l'obstruction de l'artère oculaire, qui cause des séquelles irréparables plusieurs fois. DK se manifeste également comme vascularite nécrosante, conduisant ainsi à la perte de tissu affecté (ALVES, et al., 2011).

Les complications neurologiques dues aux dommages de cns peuvent être comme suit : méningoencéphalite, collection sous-dural, hypoperfusion cérébrale, ischémie, infarctus cérébral, saisies, émigrégie, confusion mentale, léthargie, et même coma ou infarctus cérébral. De telles complications peuvent devenir irréversibles, nuissant ainsi à jamais à la vie du patient (ALVES, et al., 2011).

Le risque de complications tardives de la DK suggère la nécessité d'un suivi à long terme en plus de l'enfance (GIACCHI et al., 2014).

\section{TRAITEMENTS DISPONIBLES POUR LES PERSONNES TOUCHÉES PAR DK}

RC: 82506

Disponível em: https://www.nucleodoconhecimento.com.br/sante/maladie-dekawasaki 
Le traitement le plus indiqué est l'application d'IGIV (Immunoglobuline intraveineuse) dans une seule dose de $2 \mathrm{~g} / \mathrm{kg}$ par perfusion de 12 heures. Selon l'évolution du quaro clinique du patient, la dose d'IGIV peut être répétée ou des corticostéroïdes peuvent être ajoutés, particulièrement dans les cas réfractaires. D'autres médicaments sont recommandés, comme le cyclophosphamide, la cyclosporine et l'ulinastatine. En outre, un produit basé sur des anticorps monoclonaux contre le facteur alpha de nécrose de tumeur est recommandé, aussi bien qu'un anticorps monoclonal, connu sous le nom d'Abciximab, et qui peut traiter des anévrismes plus rapidement (ATIK, 2007; FERRONATO et al., 1010; KAYIRAN; DINDAR; GURAKAN, 2010; PHILIP et al., 2017; SOTELO; GONZÁLEZ, 2007).

Environ 10 à $15 \%$ des patients atteints de DK ne présentent aucune amélioration avec le traitement IGIV, qui est le traitement standard (MEHNDIRATTA et al., 2014). Chez les patients pédiatriques atteints de DK, des doses élevées d'acide acétylsalicylique (AAS) fournissent une action anti-inflammatoire, tandis que de faibles doses ont un effet inhibiteur de l'agrégation plaquettaire. Ainsi, dans la phase aiguë de la maladie, AAS $80-100 \mathrm{mg} / \mathrm{kg} /$ day est utilisé, qui est divisé en quatre ingestions tout au long de la journée, afin d'intensifier l'effet anti-inflammatoire de I'IGIV. Alors que dans certains hôpitaux la dose d'AAS est réduite après 2-3 jours en l'absence de fièvre, dans d'autres centres de santé le traitement persiste pendant jusqu'à 2 semaines. En outre, les enfants avec des anomalies cardiaques ont une prescription pour l'audition aas indéfiniment (ATIK, 2007; CASTRO; URBANO; COSTA, 2009; MEHNDIRATTA et al., 2014).

Des médicaments immunosuppresseurs tels que le cyclophosphamide sont également utilisés, ainsi que la prednisone ou la méthylprednisolone, pour améliorer certains symptômes, comme la fièvre, mais ces médicaments n'ont pas d'effet thérapeutique sur les anomalies cardiaques (CASTRO; URBANO; COSTA, 2009; PHILIP et al., 2017).

RC: 82506

Disponível em: https://www.nucleodoconhecimento.com.br/sante/maladie-dekawasaki 
Dans la phase aiguë de la maladie de DK, il y a un risque chirurgical plus élevé parce que, en présence de l'inflammation, l'anastomosis avec l'artère coronaire enflammée court le risque d'obstruction. L'approche chirurgicale devrait être limitée aux patients présentant des anévrismes géants ou des processus ischémiques après la phase aiguë (ATIK, 2007 ; SOTELO; GONZÁLEZ, 2007).

La revascularisation chirurgicale des lésions coronaires secondaires à DK est relativement rare. Selon les lignes directrices actuelles, la revascularisation artérielle complète devrait être utilisée chez les jeunes patients présentant de petites comorbidités afin d'obtenir une bonne perméabilité à long terme de greffe (BARCA et al., 2019).

En plus de la chirurgie de revascularisation myocardique, qui a été le traitement de choix pour les complications coronaires thrombotiques de DK, l'intervention coronaire percutanée (ICP) avec les entrailles régulières ou percutanées avec le polytetrafluoroethylène (PTFE), l'implantation de bobine, la revascularisation coronaire transluminaire percutanée (PTCR) avec des agents thrombolytiques dans l'artère infarctus-connexe, et l'ablation coronaire percutanée de rotation (PTCRA), sont employées dans les patients affectés par la maladie (MUTLUER ; ÇELIKER, 2019).

Quant à la gestion anesthésique, il y a peu d'information décrite, cependant, la revascularisation myocardique avec l'anesthésie exécutée par les gaz halogènes présente moins de dommages, montrant ainsi une meilleure récupération postopératoire de la fonction myocardique comparée aux anesthésiques intraveineux. Ainsi, il favorise l'inhibition de la réponse neuroendocrine au stress, et prépare également le patient à une extubation tôt dans la période postopératoire, qui aide à la prévention des complications possibles résultant de l'intubation (MARTíNEZ ; MÉNDEZ, 2013).

RC: 82506

Disponível em: https://www.nucleodoconhecimento.com.br/sante/maladie-dekawasaki 


\section{CONCLUSION}

L'occurrence de DK est variable, étant plus présente dans la région de l'Asie, particulièrement chez les enfants de moins de 5 ans. En Amérique latine, l'incidence est moins fréquente et la mortalité de cette pathologie est faible.

L'agent étiologique de DK n'a pas été identifié, mais il y a quelques agents pathogènes qui peuvent être liés à cette maladie. II y a des preuves que cette maladie a une relation génétique, ceci a été trouvé en raison de l'incidence accrue parmi les personnes asiatiques et parmi des parents des patients affectés par DK, en outre, il y a également quelques gènes qui sont associés à cette pathologie.

II y a des changements liés à DK tels que des inflammations systémiques, affectant certains organes provoqués par l'angiite, la méningite stérile, la pneumonite, l'adénofinite et l'hépatite. DK affecte les organes et les artères, principalement les vaisseaux de calibre moyen, tels que ceux du cœur, de sorte que conduit à la formation d'anévrismes, peut affecter les yeux, les muqueuses de la cavité buccale, le poumon, l'intestin, la vésicule biliaire et le système nerveux, le système lymphatique, le système cutané et les lésions peuvent se produire dans les lèvres.

L'image clinique de DK a trois étapes. Le stade fébrile aigu est caractérisé par la congestion conjonctivale, la mucosite orale, l'érythème, l'écaillé, l'éruption polymorphe, et la lymphadenopathy laterocervical. Le stade subaigu se produit à la fin de la fièvre, les patients ont la peau écaurant sur les membres, l'arthrite, l'arthralgie et la thrombocytose. Le stade de convalescence survient lorsque les symptômes sont presque en train de disparaître et se poursuit jusqu'à leur normalisation.

Le retard dans le diagnostic correct de DK est assez fréquent, qui peut mener à un retard dans le début du traitement, qui peut causer des complications pour aggraver

RC: 82506

Disponível em: https://www.nucleodoconhecimento.com.br/sante/maladie-dekawasaki 
l'état clinique. Le diagnostic clinique tôt est extrêmement important pour établir le traitement tôt et éviter des dommages de l'artère coronaire.

Le traitement de DK est basé sur l'évolution de l'image clinique du patient. La thérapie la plus utilisée est l'application de l'IGIV, si des changements se produisent dans la clinique, la dose d'IGIV peut être répétée ou des corticostéroïdes peuvent être ajoutés. D'autres médicaments peuvent être indiqués, comme le cyclophosphamide, la cyclosporine et l'ulinastatine. II est également recommandé un produit à base d'anticorps monoclonaux Infliximab, immunosuppresseurs tels que la cyclophosphamide ou la cyclosporine et la prednisone ou méthylprednisolone peut être utilisé. Le traitement chirurgical est fait principalement dans la phase aiguë de DK, mais devrait être limité aux patients présentant des anévrismes géants ou des processus ischémiques après la phase aiguë de la maladie.

\section{RÉFÉRENCES}

ALMEIDA, F. C. Avaliação das manifestações clínicas e achados laboratoriais em 301 pacientes com doença de Kawasaki: acompanhamento de dez anos. Tese (Doutorado em Ciências Médicas). Faculdade de Medicina, Universidade de Brasília, Brasília. 2018.

ALMEIDA, M. A. A. L. S. Avaliação da calprotectina e dos anticorpos anticitoplasma de neutrófilos como marcadores de inflamação e autoimunidade nas diferentes fases da doença de Kawasaki. Dissertação (Mestrado em Ciências Farmacêuticas). Faculdade de Ciências da Saúde, Universidade de Brasília, Brasília. 2017.

ALMEIDA, R. G. et al. Perfil da doença de Kawasaki em crianças encaminhadas para dois serviços de reumatologia pediátrica do Rio de Janeiro, Brasil. Revista Brasileira de Reumatologia, v. 50, n. 5, p. 529-538, set./out. 2010.

RC: 82506

Disponível em: https://www.nucleodoconhecimento.com.br/sante/maladie-dekawasaki 
ALVES, N. R. M. et al. Estudo prospectivo das complicações da Doença de Kawasaki: análise de 115 casos. Revista da Associação Médica Brasileira, v. 57, n. 3, p. 299-305, mar. 2011

ATIK, E. Doença de Kawasaki: Regressão de Aneurismas Gigantes das Artérias Coronárias com Obstrução Tardia Posterior Kawasaki Disease: Giant Coronary Arteries Aneurysms Regression and Later Stenosis. Arquivos brasileiros de Cardiologia, v. 88, n. 1, p. 22-23, 2007.

ATIK, E.; FORONDA, A.; BUSTAMANTE, L. N. P. Kawasaki Disease. Involution of Giant Coronary Aneurysms After Prolonged Anti-inflammatory Treatment. Arquivos brasileiros de Cardiologia, v. 81, n. 3, p. 265-72, set. 2003.

ATIK, E. et al. Caso 6/2017 - Extenso Aneurisma Gigante de Artéria Coronária Esquerda por Vasculite de Kawasaki em Homem Assintomático com 48 Anos de Idade. Arquivos brasileiros de Cardiologia, v. 109, n. 5, p. 489-490, nov. 2017.

BARCA, L. V. et al. Ligature of the Left Main Coronary Artery after Surgery in Kawasaki Disease: Case Report. Braz J Cardiovasc Surg, v. 34, n. 1, p. 111-113, jan./fev., 2019.

BRIEDÉ, S. et al. Hamoen M, Oosterveld M JS, Breur JMPJ. Langetermijneffecten van de ziekte van Kawasaki. Ned tijdschr geneeskd, v. 154, n. 2121, p. 1-6, out. 2015.

CASTRO, P. A.; URBANO, L. M. F.; COSTA, I. M. C. Doença de Kawasaki. Anais Brasileiros de Dermatologia, v. 84, n. 4, p. 317-331, mai. 2009.

CHBEIR, D. et al. Kawasaki disease: abnormal initial echocardiogram is associated with resistance to IV Ig and development of coronary artery lesions. Pediatric Rheumatology Onlinw Journal, v. 6, n. 48, p. 1-10, jul. 2018.

RC: 82506

Disponível em: https://www.nucleodoconhecimento.com.br/sante/maladie-dekawasaki 
FERRONATO, A. E. et al. Doença de Kawasaki: experiência clínica em hospital universitário. Revista Paulista de Pediatria, v. 28, n. 2, p. 148-154, jun. 2010.

GIACCHI, V. et al. Avaliação do espessamento íntimo da artéria coronária em pacientes com diagnóstico prévio de doença de Kawasaki por meio de ecocardiografia transtorácica de alta resolução: nossa experiência. BMC Cardiovasc Disord, v. 14, n. 106, p 1-6, ago. 2014.

HUANG, X. et al. Influenza infection and Kawasaki disease. Revista da Sociedade Brasileira de Medicina Tropical, v. 48, n. 3, p. 243-248, jun. 2015.

KAYIRAN, S. M.; DINDAR, A. N.; GURAKAN, B. An evaluation of children with Kawasaki disease in Istanbul: a retrospective follow-up study. Clinical Science, v. 65, n. 12, p. 1261-1265, dez. 2010.

KENTSIS, A. et al. Urine proteomics for discovery of improved diagnostic markers of Kawasaki disease. EMBO Mol Med, v. 5, n. 2, p. 210-220, fev. 2013.

KIM, J. S.; KWON, S. H. Atypical Kawasaki disease presenting a retropharyngeal abscess. Braz J Otorhinolaryngol, v. 82, n. 4, p. 484-486, 2016.

LIU, M. Y. et al. Risk factors and implications of progressive coronary dilatation in children with Kawasaki disease. BMC Pediatrics, v. 17, n. 139, p. 1-7, jun. 2017.

MAGALHÃES, C. M. E. Estudo da prevalência da perda auditiva neurossensorial como complicação da doença de Kawasaki. Tese (Doutorado em Ciências da Saúde) - Faculdade de ciências da saúde, Universidade Federal de Brasília, Brasília. 2008.

MAGALHÃES, C. M. R.; ALVES, N. R. M. Doença de Kawasaki. In: I. Burns, DAR, Campos Júnior D, Silva LR, Borges WG. Tratado de pediatria: Sociedade Brasileira de Pediatria. 4. ed. Barueri, SP: Manole, 2017. p.1825-1835.

RC: 82506

Disponível em: https://www.nucleodoconhecimento.com.br/sante/maladie-dekawasaki 
MARTÍNEZ, U. M.; MÉNDEZ, F. M. Manejo anestésico del paciente com enfermedad de Kawasaki durante la cirugía de revascularización coronaria: informe de um caso. Archivos de Cardiología de México, v. 83, n. 4, p. 267-272, abr. 2013.

MEHNDIRATTA, S. et al. A case of incomplete and refractory Kawasaki disease: Diagnostic and therapeutic challenges. The South African Journal of Child Health, v. 8, n. 1, p. 37-38, fev. 2014.

MUTLUER, F. O.; ÇELIKER, A. Comment on Ligature of the Left Main Coeonary Artery after Surgery in Kawasaki Disease: Case Report. Brazilian Journal of Cardiovascular Surgery, v. 34, n.3, p. 382, jul. 2019.

PHILIP, S. et al. Role of Antioxidants in Horse Serummediated Vasculitis in Swine: Potential Relevance to Early Treatment in Mitigation of Coronary Arteritis in Kawasaki Disease. Pediatrics and Neonatology, v. 58, n. 4, p. 328-337, fev. 2017.

PRINTZ, B. F. Noncoronary Cardiac Abnormalities Are Associated With Coronary Artery Dilation and With Laboratory Inflammatory Markers in Acute Kawasaki Disease. Journal of the American College of Cardiology, v. 57, n. 1, p. 68-92, jan. 2011.

RODRIGUES, M. et al. Doença de Kawasaki e Complicações Cardiovasculares em Pediatria. Birth and growth medical jornal, v.27, n. 1, p. 54-58, fev. 2017.

ROSSI, F. S. et al. Extensa linfadenite cervical mimetizando adenite bacteriana como primeira manifestação da doença de Kawasaki. Einstein, v. 13, n. 3, p. 426429, jul./set. 2015.

ROWLEY, A. H. et al. Allograft Inflammatory Factor-1 Links T-Cell Activation, Interferon Response, and Macrophage Activation in Chronic Kawasaki Disease Arteritis. Journal of the Pediatric Infectious Diseases Society, v. 6, n. 3, p. 94102, set. 2017.

RC: 82506

Disponível em: https://www.nucleodoconhecimento.com.br/sante/maladie-dekawasaki 
SCARDINA, G. A. et al. Oral necrotizing microvasculitis in a patient affected by Kawasaki disease. Medicina Oral Patologia Oral y Cirugia Bucal, v. 12, n. 8, p. 560-564, dez. 2007.

SOTELO, N.; GONZÁLEZ, L. A. Kawasaki disease: A rare pediatric pathology in Mexico Twenty cases report from the Hospital Infantil del Estado de Sonora. Archivos de Cardiologia de Mexico, v. 77, n. 4, p. 299-307, dez. 2007.

XIE, X. F. et al. Proteomics study of serum exosomes in Kawasaki disease patients with coronary artery aneurysms. Cardiology Journal, v. 26, n. 5, p. 584-593, fev. 2018.

YAP, C. Y.; LIN, L. H.; WANGI, N. K. An atypical presentation of Kawasaki disease: a 10- year-old boy with acute exudative tonsillitis and bilateral cervical lymphadenitis. CLINICS, v. 67, n. 6, p. 689-692, 2012.

Soumis : avril 2021.

Approuvé : avril 2021.

RC: 82506

Disponível em: https://www.nucleodoconhecimento.com.br/sante/maladie-dekawasaki 